\title{
RNA that activates transcription
}

Synthetic small RNA transcriptional activators can regulate gene transcription in Escherichia coli.

'Learn from nature and copy what it does' is one of the guiding principles in the laboratory of Julius Lucks at Cornell University, but in their recent work, the researchers developed a strategy that seemingly expands what nature has to offer.

"We want to leverage our ability to model and measure RNA structures to make gene networks," says Lucks. His team focuses on transcriptional control, and they aim to have RNA inputs control RNA outputs without involving proteins such as transcription factors. "The big conceptual advantage of RNA over proteins is that you can do design," explains Lucks. "We know a lot more about RNA folding than we do about protein folding."

The strategy of the Lucks team has been to observe RNA design principles in nature, characterize their structure and then apply these designs to the engineering of genetic circuits. The limitation is that whereas nature very efficiently uses small RNAs to repress transcription, there are to date no known instances of small RNAs alone activating transcription. "But," says Lucks, "if you want to build networks, you need to turn things on as well as off."

Melissa Takahashi, a graduate student in the lab, first focused on characterizing the function of a natural RNA transcriptional repressor mechanism: a special sequence upstream of a gene's coding region that can form RNA structures that allow or prevent progression of the RNA polymerase. These structures are switchable: in one case transcription is stopped by a transcriptional terminator RNA hairpin, and in the other case transcription is allowed by an antiterminator sequence that sequesters the terminator and prevents the formation of the blocking hairpin. Takahashi looked at the structural transitions needed in order to undergo the switch from active to inactive transcription; she then came up with a strategy to invert this repression mechanism into one that activates transcription by adding yet another layer of structural transitions using a small transcription activating RNA (STAR).

These added layers make for a complex mechanism, and James Chappell, a postdoctoral associate, sought to develop a more direct approach. He designed a STAR that contained an antiterminator that directly bound and sequestered a terminator sequence placed upstream of a gene and thus removed the hairpin and allowed transcription.

Interestingly, although Takahashi’s design was more complex, it worked immediatelyin contrast to the conceptually simpler STAR antiterminators, which needed more adjustment. Lucks recalls the challenges of getting the right length of the terminator sequence and a STAR with enough complementarity to sequester efficiently without being too long and forming undesired structures. Part of the difficulty was that most current RNA structure algorithms predict equilibrium folds, but STARs likely rely on rapid folding kinetics as they try to stop a polymerase that is transcribing at an average of 20 nucleotides per second, and far fewer tools and models can address such rapid switches in structure.

The team addressed these challenges by using kinetic models of gene expression, coupled with measurements on a library of STAR variants, to develop design principles that allowed them to achieve a STAR design that showed about 100-fold induction of transcription, which is close to the activation seen with protein-based regulators.

They then applied STARs to RNA-based logic gates in E. coli. "These gates can be strapped together like Legos in front of genes," says Lucks. They showed a twoinput AND gate in which the expression of the gene depended on the presence of the two STARs. Lucks predicts that the number of required inputs could be increased and that four-input AND gates should be possible. The advantage of RNA-based logic over protein-driven logic is a more shallow architecture to integrating signals: all RNA inputs drive a single gene, making the logic gates faster and more robust.

"It's a pretty simple thing; why have we not found it in nature?" Lucks wonders, but then adds that knowing more about how RNA-based activators can work will help in knowing where to look for them in nature. "Nature had to have figured it out. We just have not found it yet."

\section{Nicole Rusk}

RESEARCH PAPERS

Chappell, J. et al. Creating small transcription activating RNAs. Nat. Chem. Biol. 11, 214-220 (2015). 